\title{
User interface design in technology service platform of performance equipments Yanna
}

\author{
Wei Jiang, Meng Zhang, Rong Jie Shi, Yujian Jiang \\ Electronic Information Engineering School \\ Communication University of China \\ *mmad_zm@aliyun.com
}

Keywords: user interface user centered design technology service platform of deduction equipment

\begin{abstract}
The user interface is the medium between man and computer, The users take advantage of the interface which is an important part of computer system to exchange information with the computer. The design and development of the user interface accounted for a large proportion in the whole workload of technology service platform design. It's quality directly affects the performance of the application system and whether it can make users feel accurately, efficiently, easily and happily when use this platform. For most users, the user interface is all they have to understand a product. This paper introduces some basic principles and elements of UI design, especially the importance of user centered design and make research on User interface design in technology service platform of performance equipment.
\end{abstract}

\section{UI Design}

UI is abbreviation of user interface which means the relationship between users and machine. In Human Machine Interaction, there is a level called interface. From the psychological sense, the interface can be divided into two levels: feeling (visual, tactile, auditory, etc.) and emotion, this interface is the user interface (UI). Design is subject of combining internal environment and the external environment of the artificial. This combination is carried around "person". "People" is one aspect of interface design, is the subject and object of design service, And the "thing" is another aspect of the design interface which contains the objective entity, the environment and the information synthesis. Just like we see a product, a building, it will not only tell people some use functions, material textures, but also bring the cognition of traditional thinking and culture incomprehensible, scientific concept to users. The analysis of "thing" also means analyze the diversity of the interface design.

User interface design is an important part of the screen products. Interface design is a complex combination of different subjects, cognitive psychology, design science, linguistics and so on are playing an important role. There are three principles of user interface design: controlled by user, reduce memory burden, maintain interface consistently.

\section{UI Design State-Of-The-Art}

UI is one of the younger branch in today's computer science. It is a scientific product which combine two major: computer science and cognitive psychology. Meanwhile, it also absorb some scientific research finding in linguistics, ergonomics and sociology etc. Through the development of several decades, it has become one of the main subjects in studying the relationship between user and computer. Especially since the 80's, with the rapid development of software engineering and the new generation of computer technology's promotion, the design and development of interface has become the most active research direction in the field of computer science. With the development of computer technology and network technology, user interface design will develop in the following directions:

high-tech 
The revolution of information technology, has brought the huge change into the computer industry. Computers will tend to be more flat, thin type. The application of portable and Pocket PC has dramatically changed the mode of office. The input mode has been developed from a single keyboard and mouse towards to multi - channel. The track ball, touch screen, light pen, voice input appeared one by one. Multimedia technology, virtual reality and powerful visual workstations provide real, dynamic images and exciting user interface. All in the computer system have their own function which make this field more rich, colorful and varied.

\section{Natural}

In the early period, user interface is simple, the man-machine conversation is the machine language. Because of the development of hardware technology and the progress of computer graphics, software engineering, artificial intelligence, and window system, etc., the interactive principle and method of Graphic User Interface, direct manipulation, and " What You See is What You Get " are all generated and widely used which instead the old type of command mode of operation, and make the human computer interface naturally put a big step forward. However, users are not just content to display or print output information through the screen. Further requests need to through visual, auditory, olfactory, tactile and shape, gesture or password to "enter" the space environment more naturally so that to format "man-machine dialogue" and achieve the "on scene" experience.

\section{Humanization}

The style of modern design has gradually moved from function doctrine to pluralism and humanization. Today's consumers have willing to show self-awareness, personal style and aesthetic taste which requirements have been reflected in the design making products more rich and detailed. On the one hand, it is demanded that the product function is complete, efficient, and suitable to operate. On the other hand, it also meets people's aesthetic and cognitive spirit needs. At present, human-computer interaction is moving from the exact to obscure, from single channel to multi-channel and from two-dimensional interaction to 3D interaction. Develop fast, low-powered multichannel interface between user and the computer.

\section{The Factors Of User Interface Design}

\section{Interface element.}

The elements of a user interface include the main color, font color, font size, interface layout, interface interaction, interface function, and interface input and output mode. Among them, what elements effect the users' work efficiency significantly are method of input/output, interaction, function distribution. When using the command based interaction system, command name, parameters are also interface elements content, how to design commands and parameters is very important. The elements that affect the user's friendly evaluation are: color, font size, interface layout, etc. This division is not absolute, because as a whole, any element of interface does not conform to the habits of users, do not meet user requirements will reduce the user acceptance of the system, and even affect the working efficiency of the user, which may lead to the user eventually give up using this system. The design of the interface elements is to make users get aesthetic feeling, improve working efficiency as while as operate easily. At present, there are lots of research about how to choose interface elements appropriately and how to design layout comfortably, the content covers the available engineering, ergonomics, cognitive psychology, aesthetics, color theory etc.

\section{User role}

In the actual work process, when end user needs a variety of different types of systems, some complicated problems will sers' real needs, different from the objective functional requirements analysis, this one has a lot of subjectivity. Although interface designers can work in accordance with the general principle, but individual users in the different cultural background, knowledge level, personal preferences may vary interface requirements greatly. For different users, there are different requirements for the software interface, the way to express themselves are also not the same. And the user interface requirements are not like business functional requirements which are. usually easy to 
clear, well documented, and it is also difficult to use specialized tools to analyze. To investigate the user interface needs, we must first investigate their own characteristics. Make comprehensive processing about the different characteristics of user groups, and then targeted to analyze its interface needs. Therefore, user role as a concept model is drawn here. User role is a collection of many users, which can represent a user's feature and facilitate the unified description according to the user type of a certain rule. The goal of user survey is through investigation and analysis of user characteristics, single user which will can not be built model can be induced as user set, and these user set can be defined as a role model, and then give different priority levels, recording to the interface requirements. The user's needs survey and their characteristics survey, namely user role definition, often proceed simultaneously. There are many methods of investigation, such as direct communication, data statistics, focus groups, card sorting, etc. The principle of user role definition is representative, correlative system function and is conducive to the needs of the interface analysis. A user role may include a large number of user individuals, and their requirements for the interface can be defined according to a certain interface model. In a system, which system based by the user role definition can be varied. A single user can belong to different reference systems when he under different user roles, but a user role ought to represent a type of interface requirements.

\section{Demand change}

For the end user, they have particularly detailed needs about interface, availability, consistency and integration, but the lack of features. Compared with the functional features, there are some tasks to do in the need of user interface and availability which include (1)user description (2)user task (3)user interface features (4)development trend (5)user interface etc.

\section{UI Design In Performance Equipment Technology Service Platform}

This paper takes the performance equipment technology service platform as an example, building user interface according to the design idea and principle explained above. This platform is designed for three parties: equipment demand side, provide side and quality inspection experts which purpose is for their better communication. It will enrich the operating mode of the deductive equipment service industry, and increase. communication opportunity among each side. These services about performance equipment will be provided by the platform: information inquiry, specific customization, standard evaluation of the production, technology forum, etc. For example:

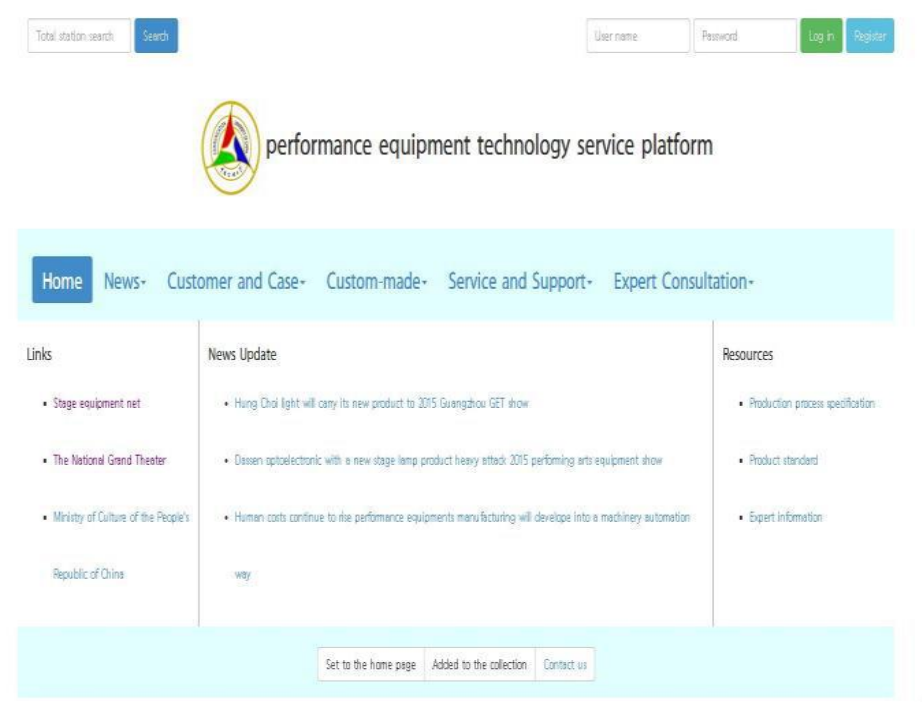

Figure 1. Performance equipment technology service platform

\section{Conclusions}


In the early period of computer's development, limited by the speed of calculation, storage capacity, and so on, users blindly pursue running speed and space who made programmers in software development have to rack their brains and consider how to make software running speed, memory / disk small. So programmers in the development of software put the main focus on data structure of the algorithm. When technology is the most attractive highlight, software interaction and interface is minor accessories. Circumstances change with the passage of time, it is the rapid development of hardware technology that made the computing speed and storage capacity become no longer a problem to worry about by developers, the main problems of concern to the user is become how to use interface easier and more comfortable. In other words, people's focus is on the ease of use and beauty about the interface, and these characteristics mainly depends on the merits of the man-machine interface. As we all know, in today's hardware and software environment, a website or software without a good interface design can not be successful. Because no matter how delicate the internal technology it have as long as the users don't want to use it, its superiority is aimless and fruitless, its value and role become a non-starter. So this field which weaken technology but emphasis ease in use and beautiful become more and more important--this is what we called UI design.

\section{Acknowledgment}

I would like to thank the National Science and Technology Support Program (Foundation project: Research on the manage systems for entertainment performance chains and key technology of web collaboration services platform, Project number: 2012BAH02F04) and Engineering Project of CUC (Founding item: Research on resources cooperative scheduling problem and optimization technique based on entertainment performance chains, Project number: 3132013XNG1305) for their help and support.

\section{References}

[1] Qian Wen, HTML + CSS web design and layout from the entry to the master, Beijing: The People's Posts and telecommunications Press ,2008

[2] XiJie LIU, Lin LIU, HTML, CSS, JavaScript web production from the entry to the master , Beijing: The People's Posts and telecommunications Press ,2013..

[3] XinQi He,Website building and web design from entry to the master , Beijing: The People's Posts and telecommunications Press ,2008.

[4] HaiYing Mi, Responsive Web Design for different devices, Suzhou Vocational University, vol 6,2013

[5] ShuMing Zhang, Design and implementation of responsive Web design, Computer and Modernization, vol 6, 2013. 